\title{
HIPPARCOS and the Calibration of the MK System
}

\author{
R.F. Garrison \\ David Dunlap Observatory, University of Toronto, Canada
}

\begin{abstract}
The HIPPARCOS satellite has provided astronomy with a wonderful legacy. We now have high-quality parallaxes for some of the yellow and red giants as well as for some of the $A$ and $F$ main sequence standards. It is now possible to compare field giants with those in globular clusters and to resolve some of the discrepancies. Hipparcos luminosities are being compared with various existing $\mathrm{MK}$ calibrations and the most glaring (i.e. interesting) inconsistencies will be discussed.
\end{abstract}

\section{Introduction}

The MK System of classifying the spectra of stars was presented in 1943 as an Atlas of Stellar Spectra by W.W. Morgan, P.C. Keenan and E. Kellman. In 1984, the methodology was generalized to include classification in general. (See Mihalas and others in Garrison 1984). The mandate of a system was defined (Garrison 1985). The MK System stands alone, independent of its calibration and of other systems, but the results are complementary and the power of the method is in the freedom from outside influences. It assumes that when different techniques, carefully used with good data, give different results, new information and insight lives at the interface between the systems.

The data from the HIPPARCOS satellite has given a giant boost to work on the calibration of the MK System. Increasing the reliability by a factor of ten or more invites a check for self consistency.

\section{Calibration of the MK Standard System}

Over the nearly sixty years since the beginnings of the MKK System, Morgan and Keenan have listed about 630 stars as MK standards. Some of these have been discarded by them for a variety of reasons. Others are difficult to reach from one or the other hemisphere. A reorganization and new calibration are underway. (See, for example, Garrison 1994.) It has been known for a long time by Morgan and his associates that the regions of the A-type dwarfs and the G-K giants were the most uncertain. These are precisely the domains opened up by HIPPARCOS.

Richard Gray's 1986 thesis addresses the problems of the A stars (see Garrison and Gray 1994 and references therein). During the course of his study, based on homogeneous, very high-quality data, it became apparent that some of the luminosity criteria used by Morgan are more sensitive to microturbulence than 
to gravity. In most cases the two are correlated, but in some cases (including a few standards) a change in the classifications was indicated. Gray's revisions are preferred.

One of Keenan's last papers carefully reconsiders the classification of the clump giants in the light of Hipparcos results. (See Keenan and Barnbaum 1999.) In letters to me, Keenan states quite clearly that he has not changed any spectral types or luminosity classes unless fully justified by the spectra themselves; i.e. unless the previous classification was clearly in error.

\section{Examples}

After investigating and comparing results for 630 standard (i.e. well classified and non-peculiar) stars, we find a fuzzy dividing line at about 5 milli-arc-seconds (mas), for values with small errors, above which there is good agreement and below which the agreement get steadily worse. The following selected examples are illustrations of the useful limits of Hipparcos data for the calibration of luminosities on the MK System.

The hottest main-sequence star for which a parallax larger than 5 mas is available is $\zeta \mathrm{Oph}$ at $09.5 \mathrm{~V}$. The $\mathrm{MK}$ type is consistent with the derived absolute magnitude of -4.25 .

On the other hand the hottest stars in NGC 2244, HD 46223 and HD 46150 $(\mathrm{O} 4 \mathrm{~V}$ and $\mathrm{O} 5 \mathrm{~V})$ have parallaxes of 0.6 mas and 2 mas respectively, yet they are at the same distance. Orion is still a bit too far for reliable parallaxes; e.g. $\delta$ and $\zeta$ Ori (O9.5 II and $\mathrm{Ib})$ are well classified as having different luminosities, but they are given different parallaxes and the same luminosity by Hipparcos. The B8 Ia supergiant $\chi^{2}$ Ori has a very small parallax yielding an absolute magnitude of -11.4 , which is not supported by the appearance of the spectra. We'll have to wait for the next generation for direct distance calibration of Orion.

There are remarkable agreements generally for stars with parallaxes larger than 5 mas and small errors. For example $\beta$ Sex, 19 Tau, and 17 Tau (B6 V, IV, and III) yield absolute magnitudes of $-0.8,-1.1$, and -1.7 from parallaxes around 9 mas with small errors. Among the cool stars, a good comparison is 61 UMa, $\xi$ Boo A, and $\beta$ Aql (G8 V, G8 V, and G8 IV) which all have parallaxes around 100 mas with small errors and which give absolute magnitudes of 5.46, 5.47 , and 2.67 , in agreement with the spectra.

The preference for Richard Gray's types for B8-F2 stars (revised before HIPPARCOS) is illustrated by the star $\alpha$ Oph. The original type is A5 III; the revised type is A5 Vnn. The parallax (70 mas) leads to an absolute magnitude of 1.29 , which is very close to Gray's value for a rapidly rotating star.

\section{Conclusion}

Hipparcos parallaxes greater than about 5 mas, with small relative errors, will be used for the calibration of the MK System; smaller parallaxes or those with large relative errors will be considered with caution. 


\section{References}

Garrison, R.F. (ed.) 1984, The MK Process and Stellar Classification (Toronto: David Dunlap Observatory, University of Toronto)

Garrison, R.F. 1985, in The Calibration of Fundamental Quantities, ed. D.S. Hayes et al. (Dordrecht: Kluwer), 17

Garrison, R.F. 1994, in The MK Process at 50 Years: A Powerful Tool for Astrophysical Insight, eds. C.J.Corbally, R.O. Gray, \& R.F. Garrison (San Francisco: ASP Conf. Ser.) 60, 3

Garrison, R.F., \& Gray, R.O. 1994, AJ, 107, 1556

Gray, R.O. 1986, Thesis, University of Toronto

Keenan, P.C. and Barnbaum, C. 1999, ApJ, 518, 859

Morgan, W.W., Keenan, P.C., \& Kellman, E. 1943, An Atlas of Stellar Spectra (Chicago: University of Chicago Press) 